\title{
Exponential-function-based droop control for islanded microgrids
}

\author{
Rui WANG ${ }^{1}$, Qiuye SUN ${ }^{1}$, , Yonghao GUI ${ }^{2}$, Dazhong MA $^{1}$
}

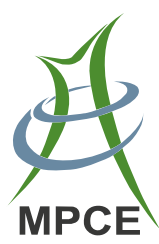

\begin{abstract}
An exponential-function-based droop control strategy for the distributed energy resources (DERs) is proposed to reduce the reactive power-sharing deviation, limit the minimum value of frequency/voltage, whilst improving the utilization rate of renewable energy. Both DERs and loads are interconnected to achieve a power exchange by converters, where the power management system should accurately share the active/reactive power demand. However, the proportional reactive power sharing often deteriorates due to its dependence on the line impedances. Thus, an exponential-function-based droop control is proposed to (1) prevent voltage and frequency from falling to the lower restraint, (2) achieve accurate reactive power sharing, (3) eliminate communication and improve the usage ratio of renewable energy. Furthermore, its stability is analyzed, and the application in islanded AC/DC hybrid microgrids is investigated to achieve the bidirectional
\end{abstract}

CrossCheck date: 26 March 2019

Received: 24 October 2018/Accepted: 26 March 2019/Published online: 10 June 2019

(C) The Author(s) 2019

$\triangle$ Qiuye SUN

sunqiuye@ise.neu.edu.cn

Rui WANG

1610232@stu.neu.edu.cn

Yonghao GUI

yg@es.aau.dk

Dazhong MA

madazhong@ise.neu.edu.cn

1 College of Information Science and Engineering, Northeastern University, Shenyang, China

2 Department of Electronic Systems, Aalborg University, Aalborg, Denmark power flow. The simulation and experimental results show that the reactive power sharing deviation can be reduced, and the utilization rate of renewable energy is improved by using the proposed method. Moreover, the simulation results illustrate that the system can maintain stable operation when the microgrid is switched from one supplied energy operation condition to another absorbed one.

Keywords Distributed energy resources (DERs), Reactive power-sharing, Droop control, Bidirectional power flow

\section{Introduction}

Recently, more and more renewable energy is integrated into smart grids or microgrids. Meanwhile, the power electronic converter has been widely used to improve the utilization of the renewable source energy [1-3]. The power electronic converter can improve not only the renewable energy usage ratio but also the power grid quality performance [4]. As a result, the power electronic converter, acting as interface equipment between distributed energy resources (DERs) and loads, is the main part of the AC/DC microgrid, and allows the elasticity of the islanded operation and the grid-connected operation $[5,6]$. Nevertheless, several power-sharing and limitation of frequency as well as voltage issues need to be investigated.

Conventionally, power sharing based on droop control strategy is broadly integrated into parallel operation of inverters [7], wherein, the active/reactive power sharing should be proportional to their rated active/reactive power that not surpassing any individual DER [8]. It is, nevertheless, not the case for reactive power sharing with the conventional droop control strategy, where impedance 
mismatch between the DERs and bus commonly causes the output voltages of inverters to be different [9]. Furthermore, frequency and voltage magnitude may fall to their lower limitation due to linear droop curve. Thus, the conventional droop control has intrinsic limitation so that the proportional reactive power sharing cannot be achieved, and frequency/voltage magnitude may fall to their lower limitation [10-12].

From the viewpoint of the limitation of frequency and voltage, the second level control was widely employed to achieve the frequency and voltage regulation [13-16]. A novel distributed coordinated control strategy based on droop coefficient via a dynamic multi-agent consensus algorithm in our previous work [13] was applied to restore the system frequency. Furthermore, in order to deal with the voltage deviation caused by the droop controller, the dynamic consensus-based distributed controller in our previous work [14] was presented to regulate the output voltages of all the DERs. Nevertheless, the communication delay may impact the performance of the system. The novel secondary voltage and frequency restoration controller was employed in [15] to assess the impact of the communication delay. However, these methods need additional communication networks, which lose the advantage of droop control strategy. Thus, the local droop controller without communication networks was proposed to achieve the voltage regulation by combining with an event-driven operator of the parameter-varying filter [16]. Meanwhile, the control strategy without using sparse communication networks, which can achieve both frequency and voltage regulations, still remains a challenge.

From the viewpoint of the reactive power sharing, there are two main approaches, i.e., droop controller with/without communication. On the side of the droop controller with communication, in [17], an adaptive virtual impedance droop control strategy based on multi-agent was proposed to make equivalent output impedance of each converter be adjusted inversely to its rated capacity. In [18], an adaptive droop coefficient control strategy was presented to realize accurate reactive power sharing. Thereinto, the excessive droop coefficients would cause the system instability, whereas low droop coefficients would affect the response speed of the system. Moreover, these above methods also need additional communication networks. Therefore, some methods without communication were developed one after another. The static virtual impedance concept was first presented to achieve reactive power sharing in the high $R / X$ ratio low-voltage microgrids [19]. Nevertheless, the performance of the reactive power sharing was not ensured on account of uncertain line impedance parameters. Therefore, some scholars put forward some improved droop controllers by adjusting droop curve feature, which is a method of nonlinear droop control strategy. In [11], a restoration mechanism based droop control strategy was first employed to improve reactive power sharing. However, this method was difficult to avoid the output voltage deviation of the converter being unable to actualize accurate reactive power sharing. Besides, the method also added the difficulty of the secondary adjustment of converter output voltage. In [20], a control strategy, the second layer control embedded in the droop controller, was first presented to realize reactive power sharing. Whilst, this method was equivalent to increasing the droop coefficient, impacting the stability of the system, and this method ignored the effect of mismatched line impedances. Thus, the improved droop control strategy without communication, which is designed to actualize accurate reactive power sharing, still also remains a problem.

Additionally, the bidirectional power flow based on droop characteristics has become an attractive problem [10]. In [8], an AC/DC consensus power management strategy was first presented to realize power interaction via the normalized AC frequency and DC voltage. Reference [21] researched the problem of active power sharing among a serious of microgrids formed by numerous AC/DC microgrids network-interconnected by interface converters. In order to deal with the aforementioned problems, this paper proposes a novel droop control strategy based on exponential function. The main characteristics and advantages of this paper are shown as follows:

1) An exponential-function-based droop control strategy without additional communication networks is proposed to achieve accurate reactive power sharing and restrict the minimum value of frequency/voltage. Furthermore, when output voltage and frequency are located far to the lower limitation in the initial stage, they can be quickly reduced to improve the utilization rate of DER by the features of exponential function.

2) According to the relationship between the frequency and the square of the voltage, this paper presents a novel AC/DC droop control strategy to realize a bidirectional power flow without additional communication networks.

This paper first proposes an exponential-function-based droop control strategy to achieve reactive power sharing accurately and restrict the frequency and amplitude of voltage in Section 2. Subsequently, the stability of the proposed droop control strategy is proved in Section 3. Moreover, a bidirectional power flow application is demonstrated to show the feasibility of the proposed droop control strategy in Section 4. Section 5 and Section 6 provide simulation and experimental results that highlight the performance of the proposed control strategy. Finally, the conclusion is obtained in Section 7. 


\section{Exponential-function-based droop control strategy}

Although the droop control method enables the converters to share the active and reactive power, there are some intrinsic limitations in this area so that proportional reactive power sharing has deviation [8]. As seen in Fig. 1, the active/reactive power flow through the lines can be represented as follows:

$$
\left\{\begin{array}{l}
P_{x}=\frac{3}{2} \frac{X_{x}}{Z_{x}} \frac{V_{x} U}{Z_{x}} \sin \phi_{x}+\frac{3}{2} \frac{R_{x}}{Z_{x}} \frac{V_{x}\left(V_{x}-U \cos \phi_{x}\right)}{Z_{x}} \\
Q_{x}=-\frac{3}{2} \frac{R_{x}}{Z_{x}} \frac{V_{x} U}{Z_{x}} \sin \phi_{x}+\frac{3}{2} \frac{X_{x}}{Z_{x}} \frac{V_{x}\left(V_{x}-U \cos \phi_{x}\right)}{Z_{x}}
\end{array} .\right.
$$

where $x=1,2 ; P_{x}$ and $Q_{x}$ are the output active and reactive power flows of the interface converter, respectively; $Z_{x}=$ $R_{x}+\mathrm{j} X_{x}$ is the equivalent impedance of DER $x ; \phi_{x}$ is the power angle of DER $x ; V_{x}$ is the voltage of DER $x$; and $U$ is the voltage at the point of common coupling. When the line impedance is pure inductive, (1) can be switched to (2).

$\left\{\begin{array}{l}P_{x}=\frac{3}{2} \frac{V_{x} U}{Z_{x}} \sin \phi_{x} \\ Q_{x}=\frac{3}{2} \frac{V_{x}\left(V_{x}-U \cos \phi_{x}\right)}{Z_{x}} .\end{array}\right.$

Thus, as shown in Fig. 2, the conventional droop control can be presented with (3) to achieve the frequency $f_{x}$ and the voltage magnitude $V_{x}$ regulation.

$\left\{\begin{array}{l}f_{x}=f^{*}-m_{x} P_{x} \\ V_{x}=V^{*}-n_{x} Q_{x}\end{array}\right.$

where $f^{*}$ and $V^{*}$ are the frequency and voltage of the droop controller without load demand; and $m_{x}$ and $n_{x}$ are the conventional droop controller coefficients. In light of (3), the accurate reactive power sharing is actualized if and only if (4) is satisfied.

$n_{1} Q_{1}-n_{2} Q_{2}=\left(V^{*}-V_{1}\right)-\left(V^{*}-V_{2}\right)=V_{2}-V_{1}=0$

Substitute (2) into (3), we have

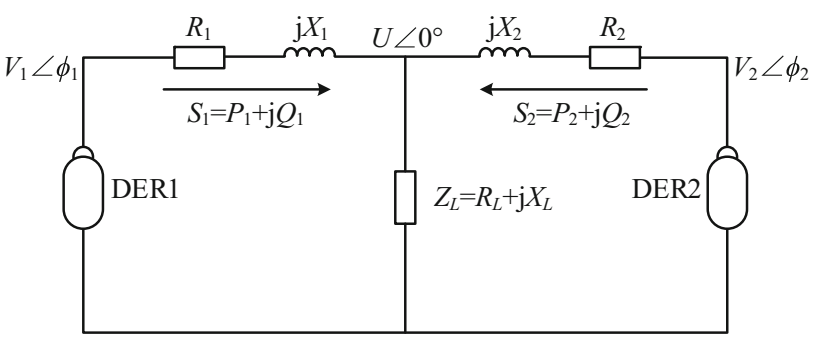

Fig. 1 Simplified circuit of the system

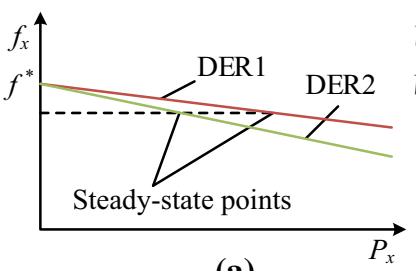

(a)

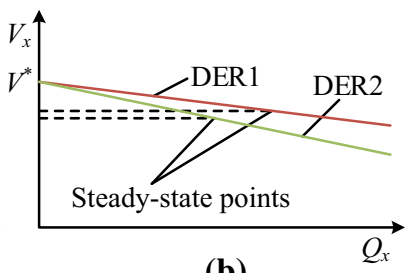

(b)
Fig. 2 Active and reactive power droop features for an AC microgrid (AMG)

$$
\begin{aligned}
V_{x}= & U \cos \phi_{x}+\frac{2}{3} \frac{Z_{x}}{n_{x}}-\left[\left(U \cos \phi_{x}\right)^{2} .\right. \\
& \left.+\left(\frac{2}{3} \frac{Z_{x}}{n_{x}}\right)^{2}+\frac{4}{3} \frac{Z_{x}}{n_{x}} U \cos \phi_{x}-\frac{8}{3} U \frac{Z_{x}}{n_{x}}\right]^{\frac{1}{2}}
\end{aligned}
$$

Furthermore, the accurate reactive power sharing is achieved if and only if (6) is satisfied.

$\left\{\begin{array}{l}\phi_{1}=\phi_{2} \\ \frac{Z_{1}}{n_{1}}=\frac{Z_{2}}{n_{2}}\end{array}\right.$

On account of the impact of line impedances, it is hard to satisfy (6) in practical system. According to the inherent characteristics of output voltage change rate of inverters, an exponential-function-based droop control strategy is proposed, as shown in Fig. 3 to overcome the effect of line impedances on reactive power sharing, where $f_{0 \min }$ and

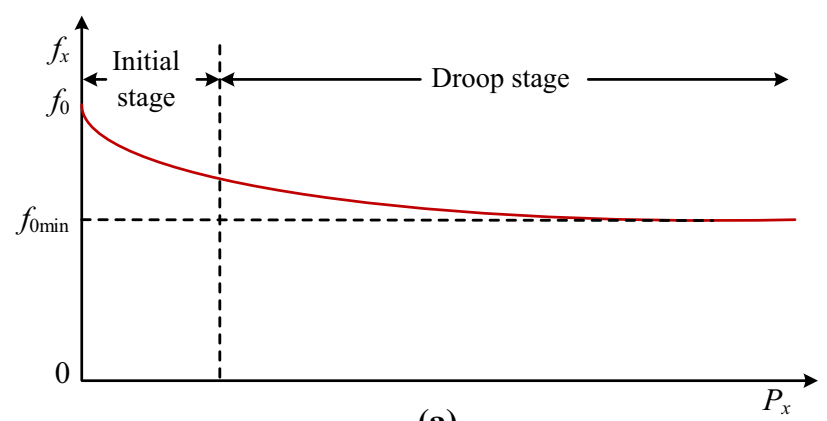

(a)

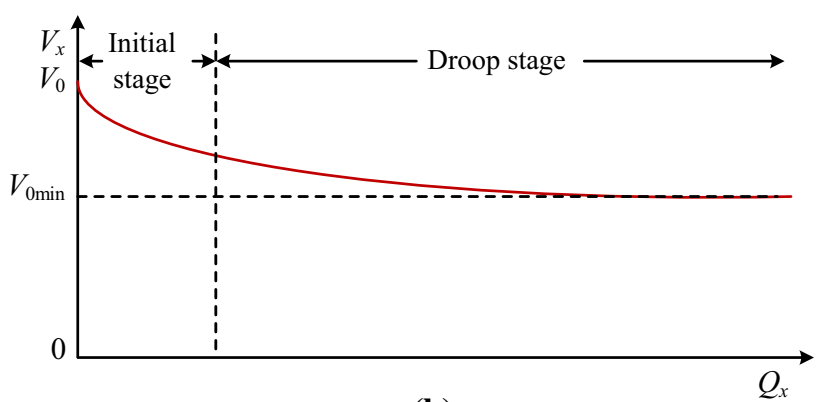

(b)

Fig. 3 A multifunctional and wireless droop control strategy based on exponential function 
$V_{0 \min }$ are the minimum values of the system, $f_{0}$ and $V_{0}$ are the no-load frequency and voltage of the converter, respectively. Compared with conventional droop control, the proposed exponential-function-based droop control strategy is a nonlinear control strategy. The controllers are described as:

$f_{x}=f_{0}-\Delta f_{x}^{*}\left(1-\mathrm{e}^{-\frac{P_{x}}{K P_{0 x}}}\right)$

$V_{x}=V_{0}-\Delta V_{x}^{*}\left(1-\mathrm{e}^{-\frac{Q_{x}}{K Q_{0 x}}}\right)$

$P_{\text {ref }}=K P_{0 x} \ln \frac{\Delta f_{x}^{*}}{f_{x}+\Delta f_{x}^{*}-f_{0}}$

$Q_{\text {ref }}=K Q_{0 x} \ln \frac{\Delta V_{x}^{*}}{V_{x}+\Delta V_{x}^{*}-V_{0}}$

where $\Delta f_{x}^{*}$ and $\Delta V_{x}^{*}$ are the rated frequency and voltage deviations of the converter, respectively; $P_{0 x}$ and $Q_{0 x}$ are the rated active power and reactive power, respectively; $P_{\text {ref }}$ and $Q_{\text {ref }}$ are the rated active power and reactive power of the inverter, respectively; and $K$ is the proportion constant. Furthermore, (11) needs to be satisfied.

$$
\left\{\begin{array}{l}
\frac{\Delta V_{x}^{*}}{K Q_{0 x}}=C_{1} \\
\Delta f_{x}^{*}=C_{2}
\end{array}\right.
$$

where $C_{1}$ and $C_{2}$ are the constant.

Remark 1 Although the linear droop control characteristic seems to be simply replaced with the exponentialfunction-based droop control characteristic, the finding process of the droop controller based on exponentialfunction is very difficult. Meanwhile, this replacement can bring many benefits. Additionally, the stability assessment of the proposed exponential-function-based droop control strategy should be studied.

To actualize the accurate reactive power sharing, the derivative of (8) is given by:

$\dot{V}_{x}=-\frac{\Delta V_{x}^{*}}{K Q_{0 x}} \mathrm{e}^{-\frac{Q_{x}}{K Q_{0 x}}}$

Figure 4 explains the managements of proposed controller in the simplified circuit shown in Fig. 1. Supposing the load is located closer to DER1, and its reactive power demand $Q_{\text {load }}$ consumption is provided by DER1 $\left(Q_{1}\left(t_{0}\right)\right)$ and DER2 $\left(Q_{2}\left(t_{0}\right)\right)$. When $Q_{\text {load }}^{0}$ increases to $Q_{\text {load }}^{1}$ at certain point, the output reactive power of DER 1 adds at express speed to $Q_{1}\left(t_{1}\right)$ in order to meet the load change demand, and the reactive power of DER2 increases marginally to $Q_{2}\left(t_{1}\right)$, since it is away from the load. Thus, the more negative $\mathrm{d} V_{1}\left(t_{1}\right)$ drives $V_{1}\left(t_{1}\right)$ lower to reduce the output reactive power of the DER1, and DER2 adds its output reactive power with its less negative $\mathrm{d} V_{2}\left(t_{1}\right)$ results

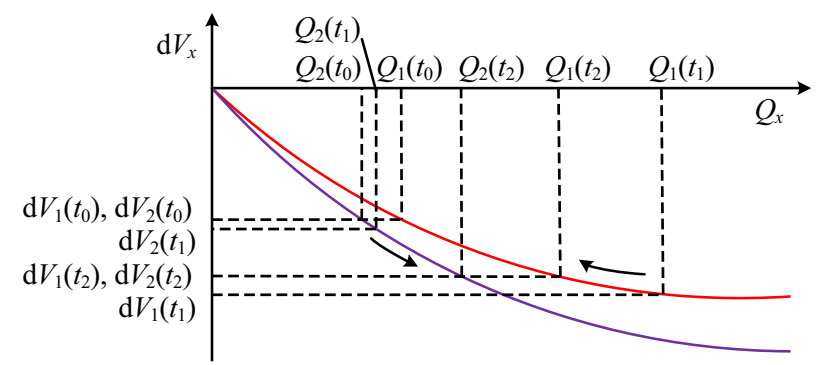

(a)

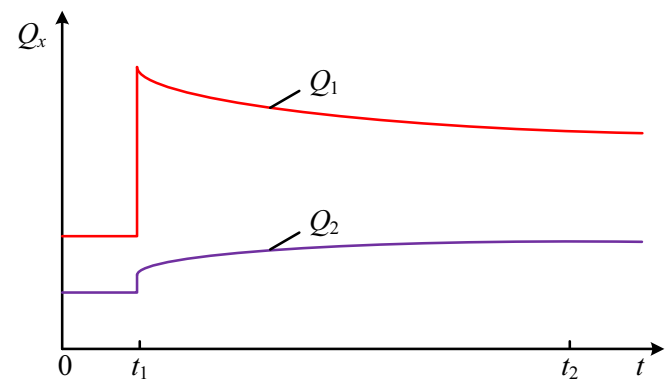

(b)

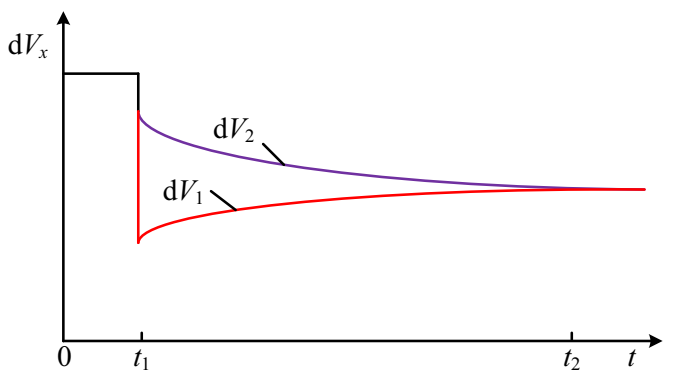

(c)

Fig. 4 Operations of proposed exponential-function-based droop controller

in relatively higher $V_{2}\left(t_{1}\right)$. Eventually, $\mathrm{d} V_{1}\left(t_{1}\right)$ and $\mathrm{d} V_{2}\left(t_{1}\right)$ are same, and the resulting $V_{1}$ and $V_{2}$ varies at the same pace, thus their output reactive power stabilize at $Q_{1}\left(t_{2}\right)$ and $Q_{2}\left(t_{2}\right)$, respectively [11].

According to the above discussion, all the DERs will operate at the identical voltage change rate in the steady state, i.e.,

$\dot{V}_{1}=\dot{V}_{2} \Rightarrow-\frac{\Delta V_{1}^{*}}{K Q_{01}} \mathrm{e}^{-\frac{Q_{1}}{K Q_{01}}}=-\frac{\Delta V_{2}^{*}}{K Q_{02}} \mathrm{e}^{-\frac{Q_{2}}{K Q_{02}}}$

The output reactive power of each DER meets the following relationship as:

$-C \mathrm{e}^{-\frac{Q_{1}}{K Q_{01}}}=-C \mathrm{e}^{-\frac{Q_{2}}{K Q_{02}}} \Rightarrow \frac{Q_{1}}{Q_{01}}=\frac{Q_{2}}{Q_{02}}$

where $C=\Delta V_{1}^{*} /\left(K Q_{01}\right)=\Delta V_{2}^{*} /\left(K Q_{02}\right)$.

Thus, the output reactive power of DERs is in coincidence with the rated reactive power capacities of DERs. In addition, the frequency of DERs is identical at the steady state shown as follows: 


$$
\begin{aligned}
& f_{1}=f_{2} \Rightarrow \\
& \quad f_{0}-\Delta f_{1}^{*}\left(1-\mathrm{e}^{-\frac{P_{1}}{K P_{01}}}\right)=f_{0}-\Delta f_{2}^{*}\left(1-\mathrm{e}^{-\frac{P_{2}}{K P_{02}}}\right)
\end{aligned}
$$

Then, from (15), we can conclude that the output active power of each DER satisfies:

$\frac{P_{1}}{P_{01}}=\frac{P_{2}}{P_{02}}$

So, the output active power of DERs is also in coincidence with the rated active power capacities of DERs. When the output voltage and frequency are located far to their lower limitation values in the initial stage, they can be quickly reduced to improve the utilization rate of renewable energy by the properties of exponential function. When the output voltage and frequency are located close to their lower limitation values, they can never fall below the lower limitation value so as to limit the voltage and frequency of the system. Thus, the proposed droop control strategy can satisfy the relevant standards better [22-24]. With conventional droop control, the frequency and voltage magnitude are always regarded as the cut-off condition. When the frequency or voltage magnitude is inferior to the minimum value, the droop control will be shifted to active/reactive power control. On account of the volatility of renewable energy, it is advisable that active power or reactive power is regarded as the cut-off condition, which can utilize the renewable energy better.

\section{Stability analysis of proposed control strategy}

The small-signal model of the conventional-functionbased droop control system has been widely investigated [25]. In this section, the steady-state function of the proposed exponential-function-based droop control is built by small-signal perturbation method. The dynamic of the power flow can be shown as follows:

$$
\left\{\begin{aligned}
\Delta P_{x} & =\frac{\partial P_{x}}{\partial V_{x}} \Delta V_{x}+\frac{\partial P_{x}}{\partial \phi_{x}} \Delta \phi_{x} \\
\Delta Q_{x} & =\frac{\partial Q_{x}}{\partial V_{x}} \Delta V_{x}+\frac{\partial Q_{x}}{\partial \phi_{x}} \Delta \phi_{x}
\end{aligned}\right.
$$

The dynamics using the small-signal approximation to linearize the equations can be expressed as:

$$
\begin{aligned}
& \left\{\begin{array}{l}
\Delta \dot{\omega}=-\omega \Delta \omega-k_{\mathrm{p}} \omega \Delta P_{x} \\
\Delta \dot{V}_{x}=-\omega \Delta V_{x}-k_{\mathrm{q}} \omega \Delta Q_{x}
\end{array}\right. \\
& \left\{\begin{array}{l}
k_{\mathrm{P}}=\frac{\Delta f_{x}}{K P_{0 x}} \mathrm{e}^{-\frac{P_{x}}{K P_{0 x}}} \\
k_{\mathrm{q}}=\frac{\Delta V_{x}}{K Q_{0 x}} \mathrm{e}^{-\frac{Q_{x}}{K Q_{0 x}}}
\end{array}\right.
\end{aligned}
$$

where $\omega$ is angle speed. Following the model approach in
[25], (20) could be given to reflect the dynamics of the entire system, which can be shown as follows:

$\boldsymbol{A}=\boldsymbol{M}_{\mathrm{s}}+\boldsymbol{C}_{\mathrm{s}}\left(\boldsymbol{I}_{\mathrm{s}}+\boldsymbol{E}_{\mathrm{s}} \boldsymbol{Y}_{\mathrm{s}}\right) \boldsymbol{K}_{\mathrm{s}}$

where

$\boldsymbol{M}_{\mathrm{s}}=\left[\begin{array}{cc}\boldsymbol{M}_{1} & \mathbf{0} \\ \mathbf{0} & \boldsymbol{M}_{2}\end{array}\right]$

$\boldsymbol{C}_{\mathrm{s}}=\left[\begin{array}{cc}\boldsymbol{C}_{1} & \mathbf{0} \\ \mathbf{0} & \boldsymbol{C}_{2}\end{array}\right]$

$\boldsymbol{I}_{\mathrm{S}}=\left[\begin{array}{cccc}i_{d 1} & i_{q 1} & 0 & 0 \\ i_{q 1} & -i_{d 1} & 0 & 0 \\ 0 & 0 & i_{d 2} & i_{q 2} \\ 0 & 0 & i_{q 2} & -i_{d 2}\end{array}\right]$

$\boldsymbol{C}_{x}=\left[\begin{array}{cc}-k_{\mathrm{p}} \omega & 0 \\ 0 & \frac{k_{\mathrm{q}} m_{q x} \omega}{m_{d x} n_{q x}-m_{q x} n_{d x}} \\ 0 & \frac{k_{\mathrm{q}} m_{d x} \omega}{m_{q x} n_{d x}-m_{d x} n_{q x}}\end{array}\right]$

$\boldsymbol{K}_{\mathrm{s}}=\left[\begin{array}{cccccc}0 & 1 & 0 & 0 & 0 & 0 \\ 0 & 0 & 1 & 0 & 0 & 0 \\ 0 & 0 & 0 & 0 & 1 & 0 \\ 0 & 0 & 0 & 0 & 0 & 1\end{array}\right]$

$\boldsymbol{E}_{\mathrm{s}} \boldsymbol{Y}_{\mathrm{s}}=\left[\begin{array}{cccc}e_{d 1} & e_{q 1} & 0 & 0 \\ -e_{q 1} & e_{d 1} & 0 & 0 \\ 0 & 0 & e_{d 2} & e_{q 2} \\ 0 & 0 & -e_{q 2} & e_{d 2}\end{array}\right]$

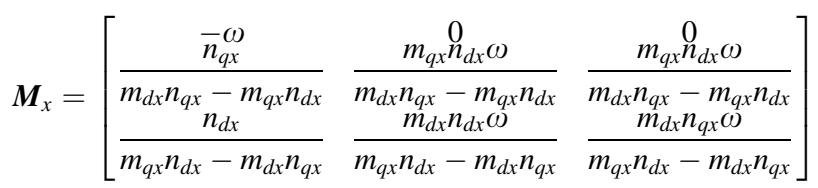

where $e_{d x}=V_{x} \cos \delta_{x} ; e_{q x}=V_{x} \sin \delta_{x} ; \delta_{x}=\arctan \left(e_{q x}\right)$ $\left.e_{d x}\right) ; m_{d x}=-e_{q x} /\left(e_{d x}^{2}+e_{q x}^{2}\right) ; m_{q x}=e_{d x} /\left(e_{d x}^{2}+e_{q x}^{2}\right) ; n_{d x}=$ $e_{d x} /\left(\sqrt{e_{d x}^{2}+e_{q x}^{2}}\right) ; n_{q x}=e_{q x} /\left(\sqrt{e_{d x}^{2}+e_{q x}^{2}}\right) ;$ and $i_{d x}, i_{q x}$ are the $d-q$ axis output currents of the inverter. In order to guarantee the stability of the proposed exponentialfunction-based droop control strategy, an eigenparticipation analysis is finished by using the linearized model to assess the small-signal stability of the system. It is clear that the system has positive eigenvalues that means the system is unstable and vice versa. The root locus is shown in Figs. 5 and 6 by changing $k_{\mathrm{p}}$ and $k_{\mathrm{q}}$, respectively. System parameters are as follows: input voltage is $400 \mathrm{~V}$; filter inductance and capacitance are $0.02 \mathrm{H}$ and $0.185 \mathrm{mF}$, respectively; capacitance of DC capacitor is $5 \mathrm{mF}$; the proportional and integral parameters of current inner loop $K_{p i}$ and $K_{i i}$ are 10.5 and 16000 , respectively; the 


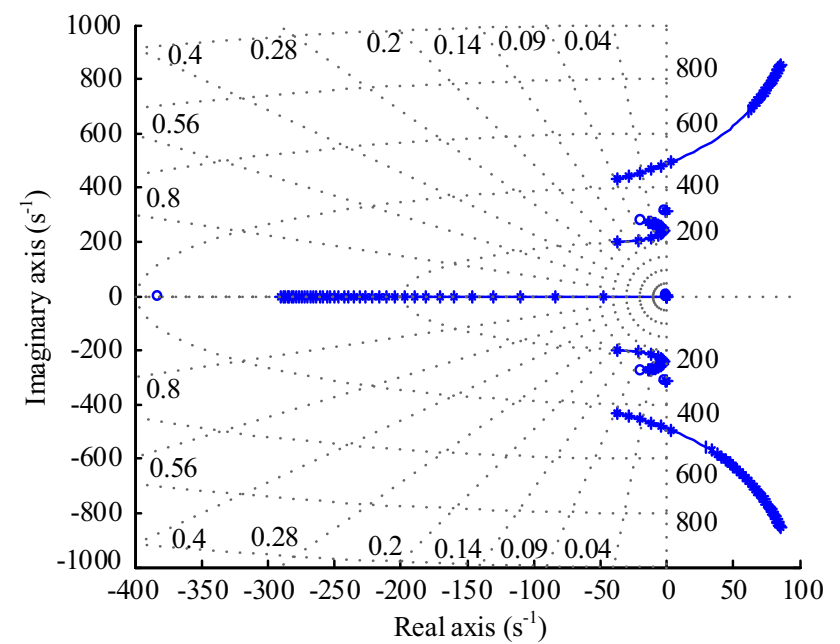

Fig. 5 Root locus of system dynamic model with $k_{\mathrm{p}}$ changing from $10^{-5}$ to $10^{-2}$

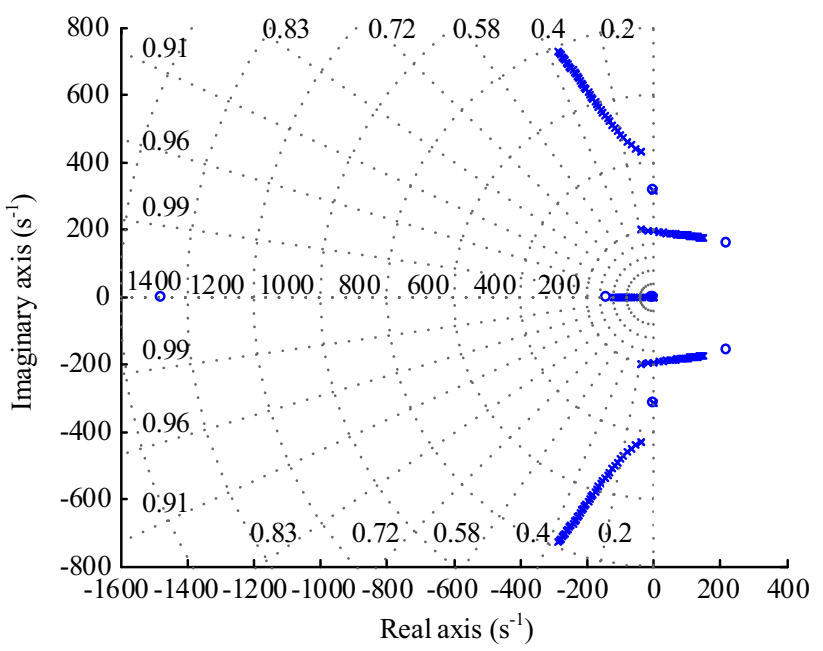

Fig. 6 Root locus of system dynamic model with $k_{\mathrm{q}}$ changing from $10^{-5}$ to $10^{-2}$

proportional and integral parameters of voltage inner loop $K_{p v}$ and $K_{i v}$ are 0.05 and 390 , respectively; $P_{0}$ is $20 \mathrm{~kW} ; Q_{0}$ is $10 \mathrm{kvar}$; active and reactive droop coefficients $k_{\omega}$ and $k_{d c}$ are 11.45 and 2, respectively; system frequency is $50 \mathrm{~Hz}$; load resistance, inductance and capacitance are $50 \Omega, 0.01$ $\mathrm{H}, 0.1 \mathrm{mF}$, respectively; frequency and voltage deviations of the converter $\Delta f_{x}$ and $\Delta V_{x}$ are 0.2 and 2, respectively; and $K$ is 1. As shown in Fig. 5, the proposed control strategy is stable if and only if the number of the right-hand planes (RHPs) of the minor loop gain is equal to zero. Thus, the proposed control strategy is stable with $k_{\mathrm{p}}$ changing from $10^{-5}$ to 0.0083 , while the value $k_{\mathrm{q}}$ is $10^{-5}$. As shown in Fig. 6, the proposed droop control strategy is stable if and only if $k_{\mathrm{q}}$ changes from $10^{-5}$ to 0.0013 , while the value $k_{\mathrm{p}}$ is $10^{-5}$. Since $k_{\mathrm{p}}$ and $k_{\mathrm{q}}$ are in stable interval, the proposed control strategy is stable. Furthermore, the stability margin of the proposed control strategy can be improved by adding $K$.

$$
\left\{\begin{array}{l}
k_{\mathrm{p}}=\frac{\Delta f_{x}}{K P_{0}} \mathrm{e}^{-\frac{P_{x}}{K P_{0}}} \in\left(1.353352832366 \times 10^{-6}, 1 \times 10^{-5}\right) \\
k_{\mathrm{q}}=\frac{\Delta V_{x}}{K Q_{0}} \mathrm{e}^{-\frac{Q_{x}}{K Q_{0}}} \in\left(2.7067056 \times 10^{-5}, 2 \times 10^{-4}\right)
\end{array} .\right.
$$

\section{Application of proposed control strategy}

With the development of the islanded AC/DC hybrid microgrids, the AC/DC droop control strategy should be proposed to achieve the bidirectional power flow. Meanwhile, the main application of the proposed control strategy is applied to AMGs. Furthermore, the application of the proposed control strategy is also extended in AC/DC hybrid microgrids. Thus, the application of the proposed control is discussed in this section, which is the bidirectional power flow. Normally, the AMG and DC microgrid (DMG) are connected through an island interconnection device (IID). The AMG is interfaced with its neighboring DMG through an interlinking converter (IC), as shown in Fig. 7, which can be regarded as a voltage source converter (VSC) [26].

In contrast to the AMG or DMG, the power management of the IC needs to bring forward a novel control strategy to manage the bidirectional power flow between AMGs and DMGs. Besides, to eliminate fast communication modes, the proposed droop control is applied to the novel AC/DC droop control strategy to achieve bidirectional power flow. The frequency of the AMG and the DC bus voltage of the IC should be measured as input signal to power management strategy to provide the power reference value [10]. As shown in Fig. 8, the stored energy in the capacitor is:

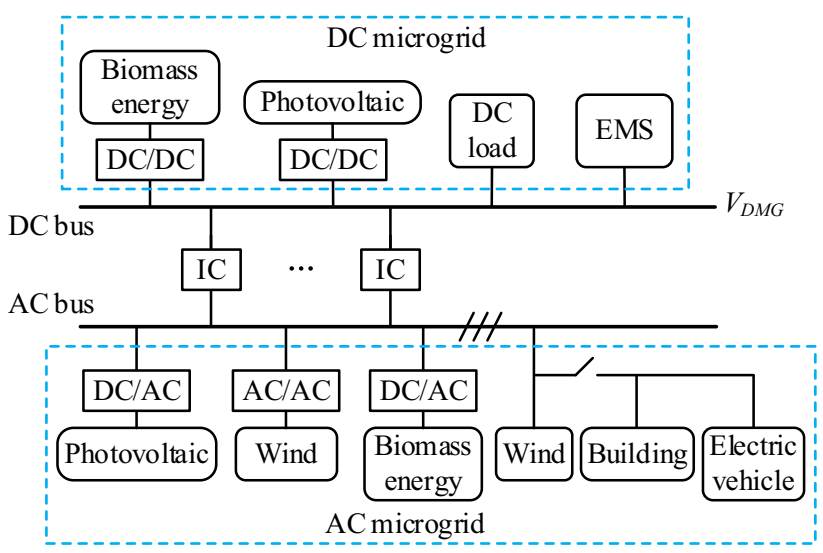

Fig. 7 A typical hybrid AC/DC microgrid 


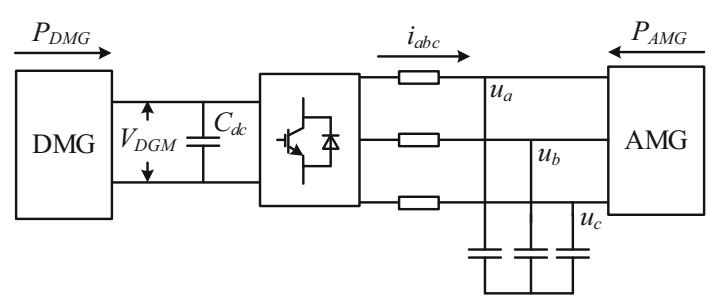

Fig. 8 Structure of IC interfacing AMG and DMG

$W_{d c}=\frac{1}{2} C_{d c} V_{D M G}^{2}$

where $W_{d c}$ is the stored energy in the capacitor; $C_{d c}$ is DC capacitor; and $V_{D M G}$ is the DC voltage of the DMG. Neglecting the switching losses converter, the dynamics in the capacitor energy is the difference of power transfer between AMG and DMG. Thus,

$\frac{\mathrm{d}}{\mathrm{d} t} W_{d c}=\frac{1}{2} C_{d c} \frac{\mathrm{d}}{\mathrm{d} t} V_{D M G}^{2}=P_{D M G}-P_{A M G}=\Delta P_{H}$

where $P_{D M G}$ and $P_{A M G}$ are output active power of the DMG and AMG, respectively; and $\Delta P_{H}$ is the active power deviation of the microgrid. In consideration of the characteristic of droop control strategy in the AMG and DMG, the related formulations are shown as follows:

$\Delta \omega_{A M G}=\omega_{A M G 0}-\omega_{A M G}=k_{\omega}\left(\mathrm{e}^{-\frac{P_{A M G 0}}{K P_{A M G 0 x}}}-\mathrm{e}^{-\frac{P_{A M G x}}{K P_{A M G 0 x}}}\right)$

$P_{D M G, \text { ref }}=\frac{1}{k_{d c}}\left(V_{D M G}-V_{D M G 0}\right)+P_{D M G 0}$

$k_{d c}=\frac{V_{D M G, \max }-V_{D M G, \text { min }}}{P_{D M G, \max }}$

where $\omega_{A M G}$ is angular velocity of the AMG; $\Delta \omega_{A M G}$ is the angular velocity deviation of the AMG; $\omega_{A M G 0}$ is no-load angular velocity of the AMG; $P_{A M G O}$ is the rated output active power of AMG; $P_{A M G 0 x}$ is the rated output active power of AMGx; $P_{A M G x}$ is the output active power of AMG $x ; P_{D M G, \text { max }}$ and $P_{D M G, \text { ref }}$ are the maximum and rated active power in the DMG, respectively; $P_{D M G 0}$ is no-load active power in the DMG; $V_{D M G 0}$ is no-load voltage of the DMG; and $V_{D M G, \text { max }}$ and $V_{D M G \text {,min }}$ are the maximum and minimum permitted voltage of the DMG, respectively.

In accordance with (30) and (31), a novel AC/DC droop control strategy for the IC is proposed through using the Taylor series expansion up to $(\rho+\gamma)$ and assuming that the frequency in the microgrid is constant, where $\gamma$ denotes the control order, and $\rho$ denotes the relative degree of the system. For the system under investigation, it is enough obvious that $\gamma$ is equal to one, whilst $\rho$ is equal to zero [27]. The droop control of the IC is given by:
$\left\{\begin{array}{l}\omega_{A M G 0}-\omega_{A M G}=k_{\omega}^{*}\left(V_{D M G 0}^{2}-V_{D M G}^{2}\right) \\ k_{\omega}^{*}=\frac{1}{2} k_{\omega} \frac{C_{d c}}{T_{s}} \frac{1}{K P_{A M G 0 x}}\end{array}\right.$

where $T_{s}$ is the sampling period; and $k_{\omega}^{*}$ is the coefficient of AC/DC droop control.

The equation of $\mathrm{AC} / \mathrm{DC}$ droop control is:

$\omega_{A M G}=k_{\omega}^{*} V_{D M G}^{2}+\left(\omega_{A M G 0}-k_{\omega}^{*} V_{D M G 0}^{2}\right)$

The AC/DC droop control strategy is exhibited in Fig. 9, where $\omega_{\min }$ is the minimum value of the angle speed in AMG. A system block diagram of the proposed interlinking bidirectional power converter control is depicted in Fig. 10. The responses of IC in different islanded modes are shown as follows.

1) Mode $1\left(\omega_{A M G}<\omega_{\min }\right.$ and $\left.V_{D M G}^{2}<V_{D M G, \text { min }}^{2}\right): P_{\text {ref }}=0$ and IC transfers no power. Both DMG and AMG are overloading. An overloading blocking logic shown in Fig. 11a is adopted to satisfy mode 1 .

2) Mode $2\left(\omega_{A M G}>\omega_{\min }\right.$ and $\left.V_{D M G}^{2}<V_{D M G \text {,min }}^{2}\right)$ : $P_{\text {ref }}=$ $P_{A M G \text {,ref }}$ and IC supplies power to the DMG, where $P_{A M G \text {,ref }}$ is the rated active power of the AMG. An IC transfer opening logic shown in Fig. $11 \mathrm{~b}$ is taken to satisfy mode 2.

3) Mode $3\left(\omega_{A M G}<\omega_{\min }\right.$ and $\left.V_{D M G}^{2}>V_{D M G \text {,min }}^{2}\right): P_{\text {ref }}=$ $-P_{D M G \text {,ref }}$ and IC supplies power to the AMG. An IC transfer opening logic shown in Fig. 11b is employed to satisfy mode 3 .

4) Mode $4\left(\omega_{A M G}>\omega_{\min }\right.$ and $\left.V_{D M G}^{2}>V_{D M G \text {,min }}^{2}\right): P_{\text {ref }}=$ 0 and IC transfers no power. A light load blocking logic exhibited in Fig. 11c is utilized to satisfy mode 4.

The rated reactive power control of the IC is simple as the reactive power does not exist in DMG, and the IC is studied to share the constant reactive power during IC

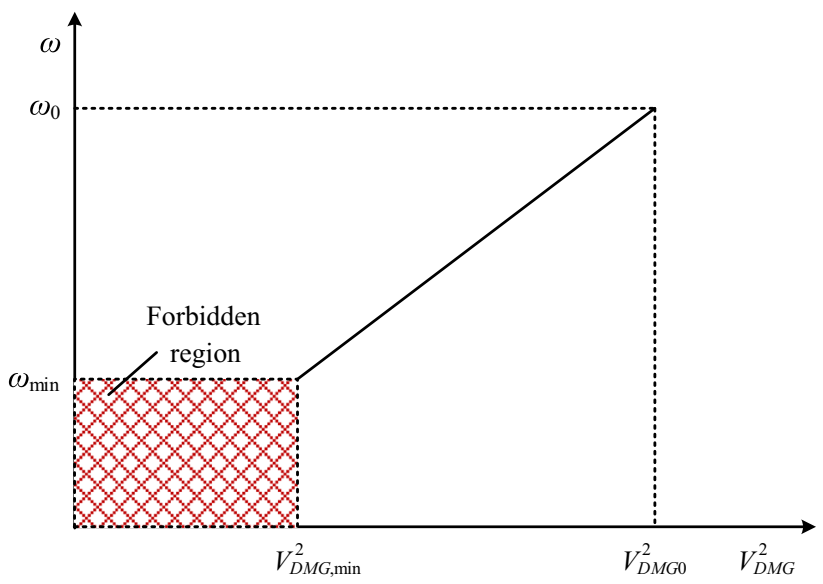

Fig. 9 A novel AC/DC droop control strategy for IC 


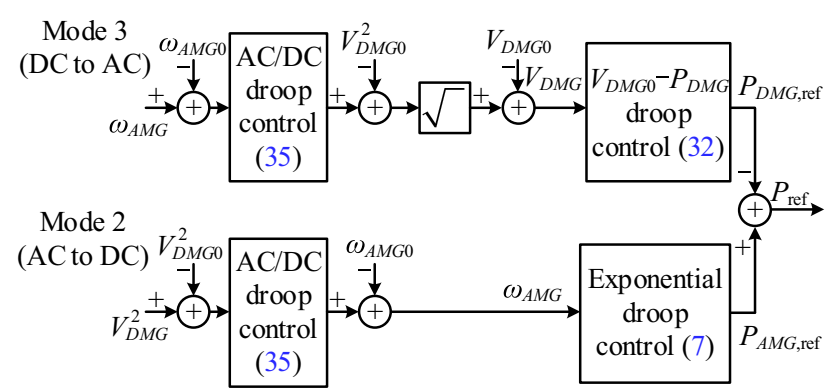

Fig. 10 Proposed interlinking bidirectional power converter control strategy

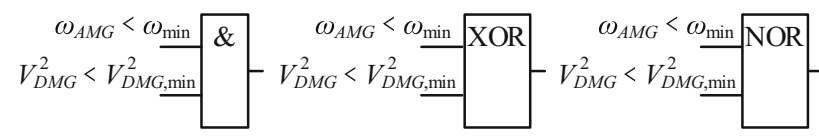

(a)

(b)

(c)

Fig. 11 Mode discrimination logic

supplying power to the AMG, the rated reactive limitation is defined as:

$Q_{I C, \max }=\sqrt{S_{I C, \max }^{2}-P_{I C}^{2}}$

where $Q_{I C \text {, max }}$ is the maximum reactive power transfer between AMG and DMG through the IC; $S_{I C \text {, max }}$ is the maximum rating of the $\mathrm{IC}$; and $P_{I C}$ is the power transfer between AMG and DMG through the IC.

\section{Simulation results}

To verify the proposed droop control strategy, a hybrid AC/DC microgrid system with multi-DERs, as shown in Figs. 1 and 8, is simulated in Simulink. System parameters are the same as those in Section 3. Different capacities of the DERs and configuration of the line impedances are designed with identical parameters to accelerate the observation of the reactive power sharing accuracy and improvement of the utilization rate of renewable energy. Eventually, the bidirectional power flow is verified.

\subsection{Case 1}

DER1 and DER2 are both DC sources with the same capacities, and both of them provide energy to the load, and the load is located closer to DER1. Furthermore, the line impedances are set as $Z_{1}=(17.5+\mathrm{j} 0.2) \mathrm{m} \Omega$ and $Z_{2}=(26.25+\mathrm{j} 0.3) \mathrm{m} \Omega$, the line load is set as $P_{\text {load }}=$ $30 \mathrm{~kW}, Q_{\text {load }}=16 \mathrm{kvar}$ and $K=1.5$. As shown in Fig. 12, compared with the sharing errors of the DERs with the conventional control strategy, the output reactive power of DER1 with the proposed control strategy is similar to that of DER2 with the proposed control strategy. Therefore, the proposed control strategy is able to reduce the reactive power sharing deviation among multi-DERs. Furthermore, the output voltages of DERs are also similar while the rated reactive power of DERs are identical.

\subsection{Case 2}

DER1 and DER2 are both DC sources, and their capacities are set as $P_{01}=20 \mathrm{~kW}, Q_{01}=10 \mathrm{~kW}$, $P_{02}=10 \mathrm{~kW}, Q_{02}=5 \mathrm{~kW}$. The load is located closer to DER1. Moreover, the line impedances are set as $Z_{1}=$ $(17.5+\mathrm{j} 0.2) \mathrm{m} \Omega$ and $Z_{2}=(26.25+\mathrm{j} 0.3) \mathrm{m} \Omega$, the line load is set as $P_{\text {load }}=25 \mathrm{~kW}, Q_{\text {load }}=12 \mathrm{kvar}$ and $K=1.5$. As shown in Fig. 13, the output reactive power of DER1 with the proposed control strategy is twice than that of DER2 with the proposed control strategy, and the sharing errors of the DERs can be reduced. Thus, the proposed control strategy is able to reduce the reactive power sharing deviation among multi-DERs. The output reactive power proportion of DERs is similar to their rated reactive power proportion.

\subsection{Case 3}

The capacities of DER1 and DER2 are set as $P_{01}=2 \mathrm{~kW}, Q_{01}=1 \mathrm{kvar}, P_{02}=20 \mathrm{~kW}, Q_{02}=10 \mathrm{kvar}$. The load is located closer to DER1, and the line load is set as $P_{\text {load }}=15 \mathrm{~kW}, Q_{\text {load }}=6 \mathrm{kvar}$. The other parameters are the same as those in case 2 . The above-mentioned case is the strictest. As shown in Fig. 14a, b, the stability of the microgrid can be satisfied in the steady state, whereas the transient output active power and reactive power of DER1 are both beyond the rated capacities. Thus, this case can also be considered that the microgrid is instantaneously unstable. Since the capacitor and inductance can store energy, the instantaneous overload energy can be provided by the capacitor and inductance. In real systems, the ability of overcurrent/overvoltage for insulated gate bipolar transistor (IGBT) is better than the rated capacity. Thus, the microgrid cannot be stable in the steady state, when transient output active power or reactive power of DER is beyond the rated capacity. As shown in Fig. 14c, d, the accurate reactive power sharing with the conventional droop control cannot be achieved, and the output reactive power of DER1 is beyond the rated capacity in the steady state. In order to ensure the stability of the system, the droop control strategy should be shifted to the active/ reactive power control in this extreme case. 


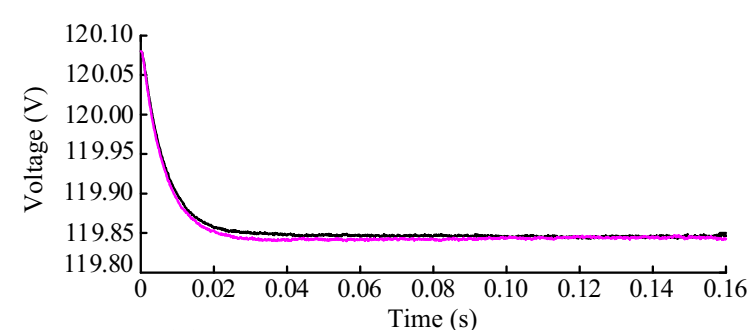

(a) Voltage with proposed exponential droop control

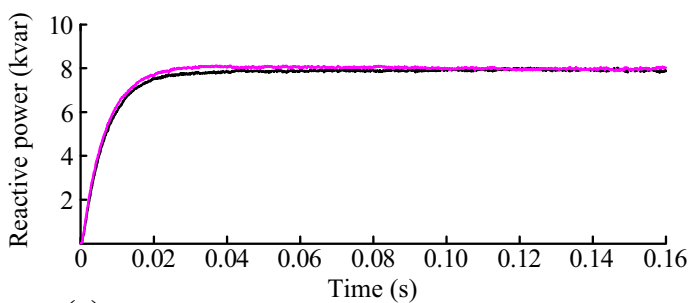

(c) Reactive power with proposed exponential droop control

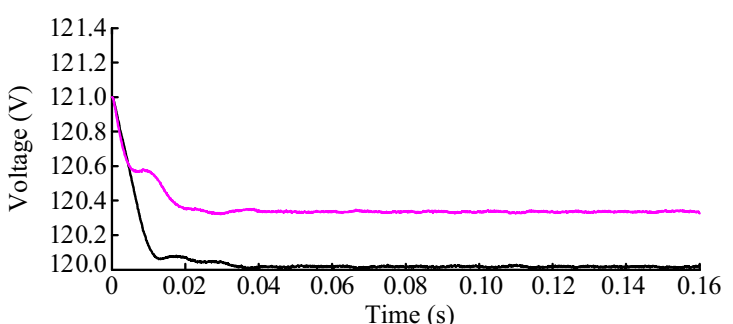

(b) Voltage with conventional droop control

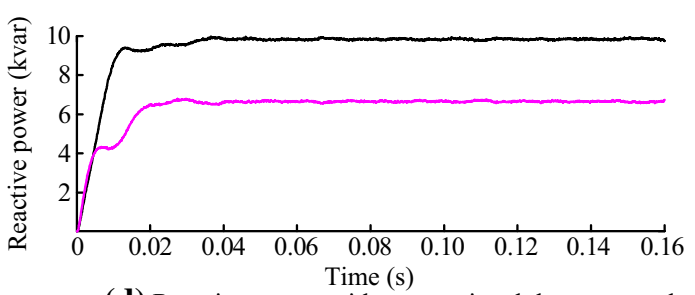

(d) Reactive power with conventional droop control

$$
\text { — DER } 1 ; \text { - DER2 }
$$

Fig. 12 Simulation results of voltage and reactive power sharing in case 1

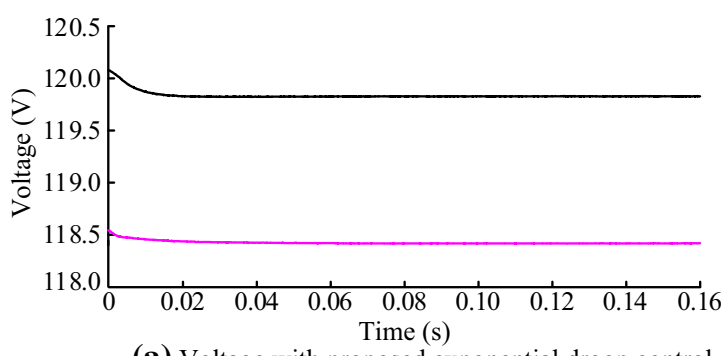

(a) Voltage with proposed exponential droop control

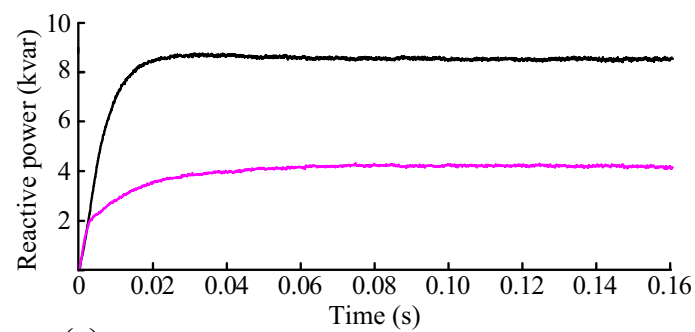

(c) Reactive power with proposed exponential droop control

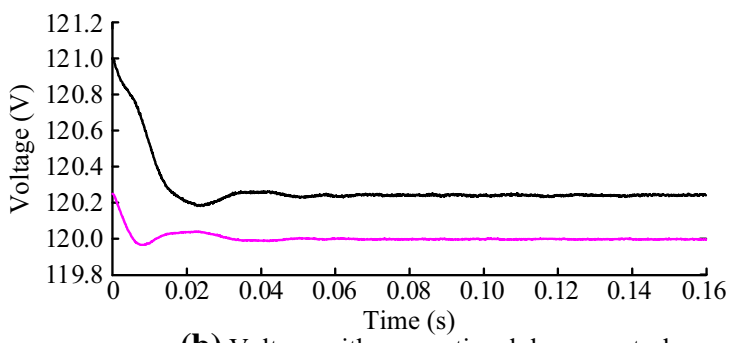

(b) Voltage with conventional droop control

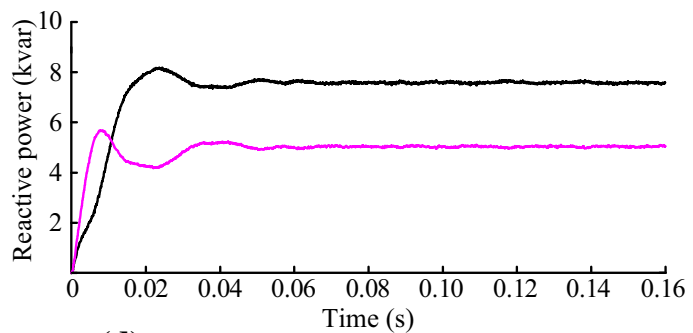

(d) Reactive power with conventional droop control

$$
\text { - DER } 1 ; \text { - DER2 }
$$

Fig. 13 Simulation results of voltage and reactive power sharing in case 2

\subsection{Case 4}

DER1 adopting conventional droop control and DER2 adopting proposed droop control are both DERs with same capacities, and both of them provide energy to the load. The line impedances are set as $Z_{1}=Z_{2}=(17.5+\mathrm{j} 0.2) \mathrm{m} \Omega$, and the line load is set as $P_{\text {load }}=30 \mathrm{~kW}, Q_{\text {load }}=16 \mathrm{kvar}$. As shown in Fig. 15, the DER employing exponential-function based droop control strategy can be integrated into the DERs which adopt conventional droop control strategy. Furthermore, the utilization rate of renewable energy is improved, when renewable energy adopts conventional droop control strategy and other energy employs proposed droop control strategy.

\subsection{Case 5}

The classic microgrid test system consisting of three DERs is provided to verify the performance of the proposed droop control strategy, which is shown in Fig. 16. Meanwhile, the rated ratio of the reactive power of the three DERs is 4:3:2. The proposed control strategy is 


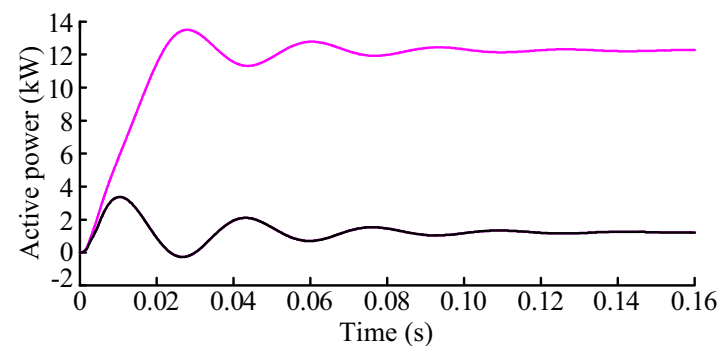

(a) Active power with the proposed droop control

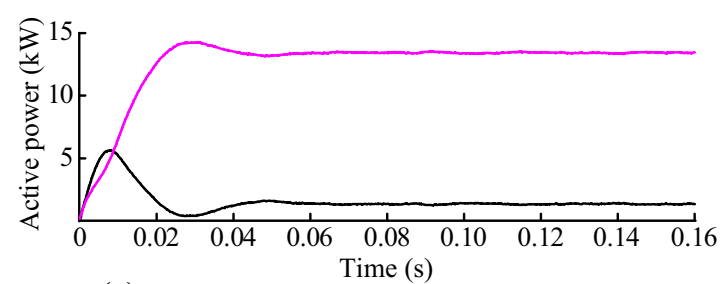

(c) Active power with the conventional droop control

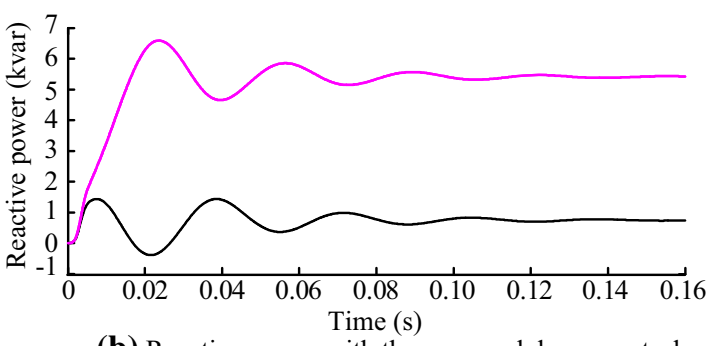

(b) Reactive power with the proposed droop control

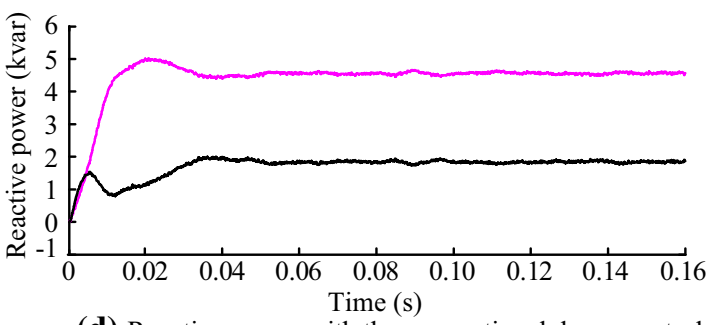

(d) Reactive power with the conventional droop control

$$
\text { — DER } 1 ; \text { - DER2 }
$$

Fig. 14 Simulation results of active power and reactive power in case 3 where one of the DERs is with low power

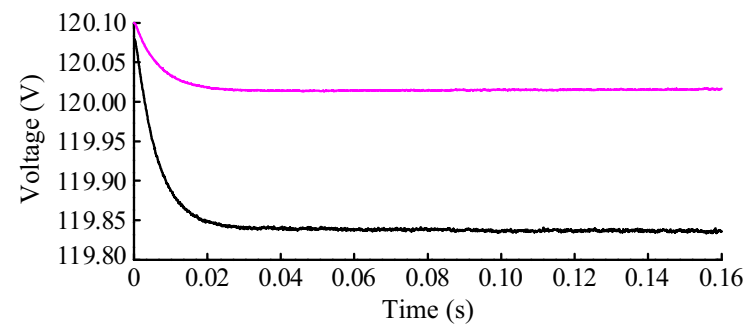

(a) Voltage

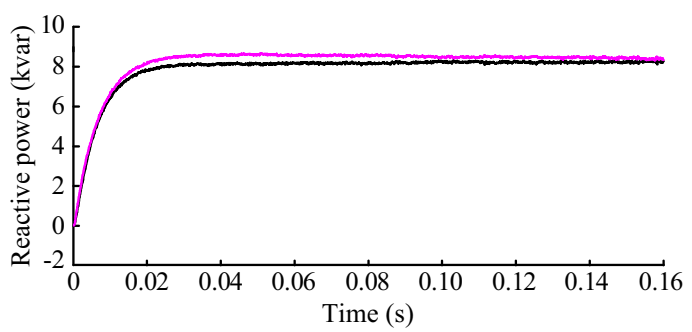

(c) Reactive power

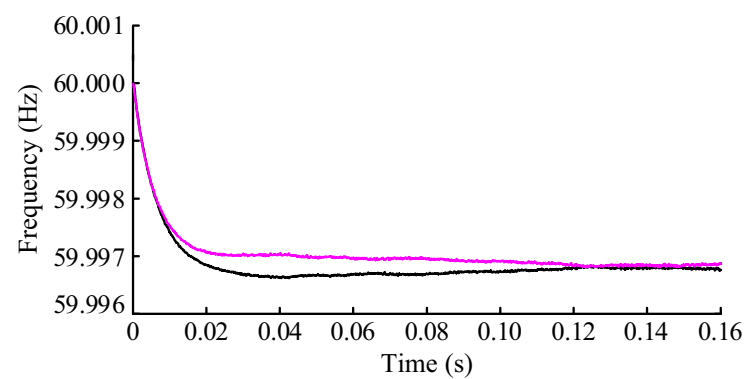

(b) Frequency

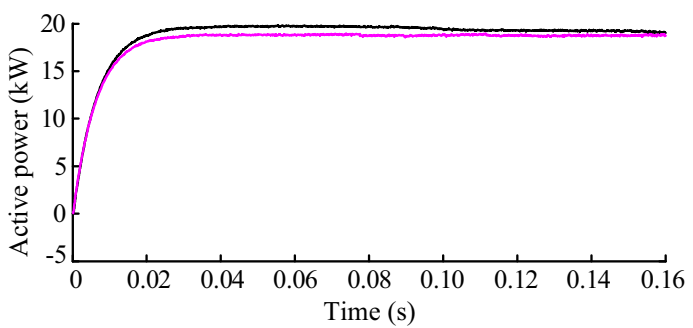

(d) Active power

- DER $1 ;$ - DER2

Fig. 15 Simulation results of voltage, frequency, reactive power, and active power in case 4 when DER1 adopts proposed exponential droop control and DER2 adopts conventional droop control

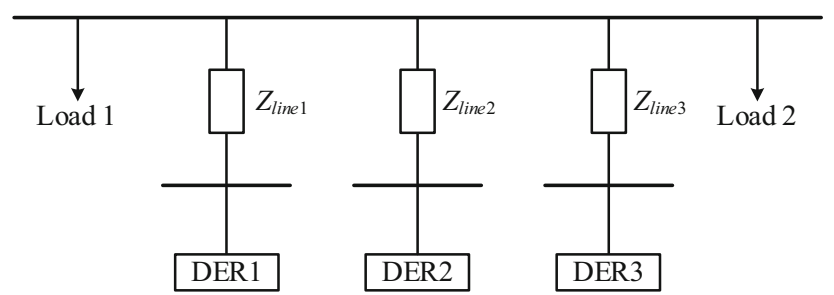

Fig. 16 Classic microgrid test system with high capacity of DERs simulated with the parameters exhibited in Section 3. Moreover, the line impedances are set as $Z_{\text {line } 1}=$ $(17.5+\mathrm{j} 0.2) \mathrm{m} \Omega, \quad Z_{\text {line } 2}=(20+\mathrm{j} 0.25) \mathrm{m} \Omega, \quad Z_{\text {line } 3}=$ $(26.25+\mathrm{j} 0.3) \mathrm{m} \Omega$, and the line load is set as $P_{\text {load }}=$ $25 \mathrm{~kW}, Q_{\text {load }}=18 \mathrm{kvar}$ and $K=1.5$. As shown in Fig. 17, the accurate reactive power sharing can be achieved by the dynamic virtual impedance [17] and the proposed droop control strategy. The performance of four droop controllers can be summarized as shown in Table 1 . In the viewpoint of the droop controller without the extra 


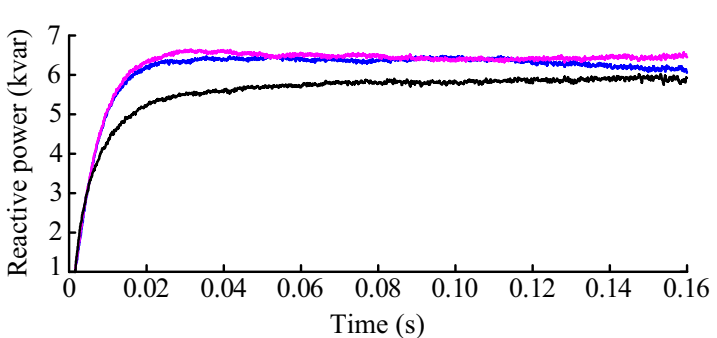

(a) Conventional droop control

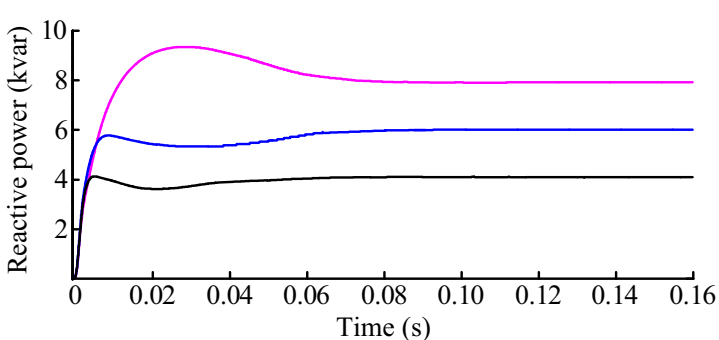

(c) Dynamic virtual impedance

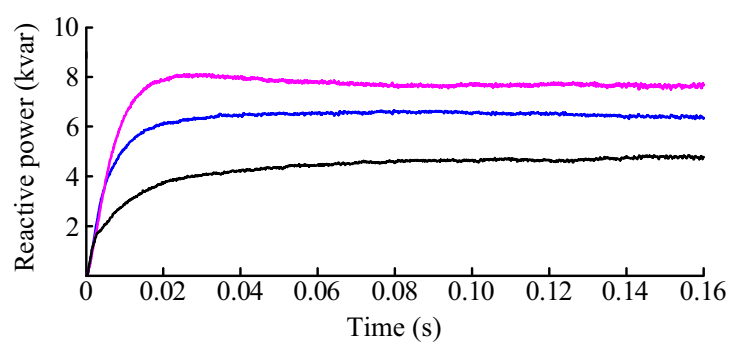

(b) Static virtual impedance

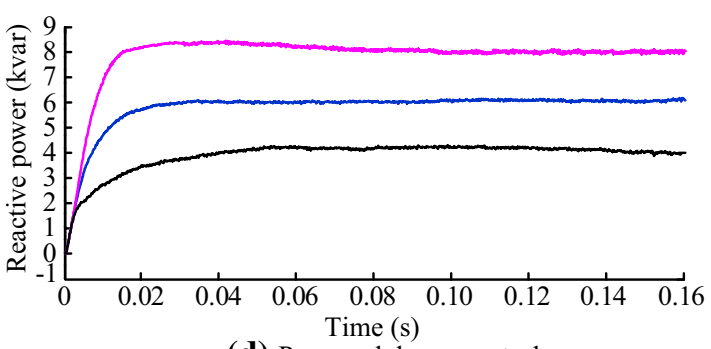

(d) Proposed droop control

Fig. 17 Reactive power simulation results in case 5

Table 1 Performance comparison analysis

\begin{tabular}{lll}
\hline Method & Communication & Performance \\
\hline Conventional droop control & No & Low \\
Static virtual impedance [19] & No & Middle \\
Dynamic virtual impedance [17] & Yes & High \\
The proposed droop control & No & High \\
\hline
\end{tabular}

communication, the performance of the proposed droop control method is better than that in [19]. In the viewpoint of the droop controller with the extra communication, the performance of the proposed droop control method is similar to that in [17]. Nevertheless, since loss of packet, communication delay and failures of communication modes will also impact the performance of the system, the reliability of the droop controller with the dynamic virtual impedance is reduced due to the extra communication networks. Thus, the effectiveness of the proposed droop control strategy is verified.

\subsection{Case 6}

DC renewable sources provide a fixed power in DMG. One part of the required load is provided via the local sources in DMG and another inadequate power is supplied via the IC at first. At $0.1 \mathrm{~s}$, a large part of the DC loads is switched off, and the DC power sources are more than the load demands. The IC switches to the inverter mode, and DMG feeds the redundant power to the AMG. The system response is exhibited in Fig. 18. It can be seen that a certain percentage of the load power requirement in the AMG is provided via the IC at first. Then, a fixed percentage of the load power requirement in the DMG is supplied through the IC at $0.1 \mathrm{~s}$. The power flow, current injection by the IC and the DC bus voltage reflect a stable system operation. Thus, this proposed control strategy is able to achieve bidirectional power flow.

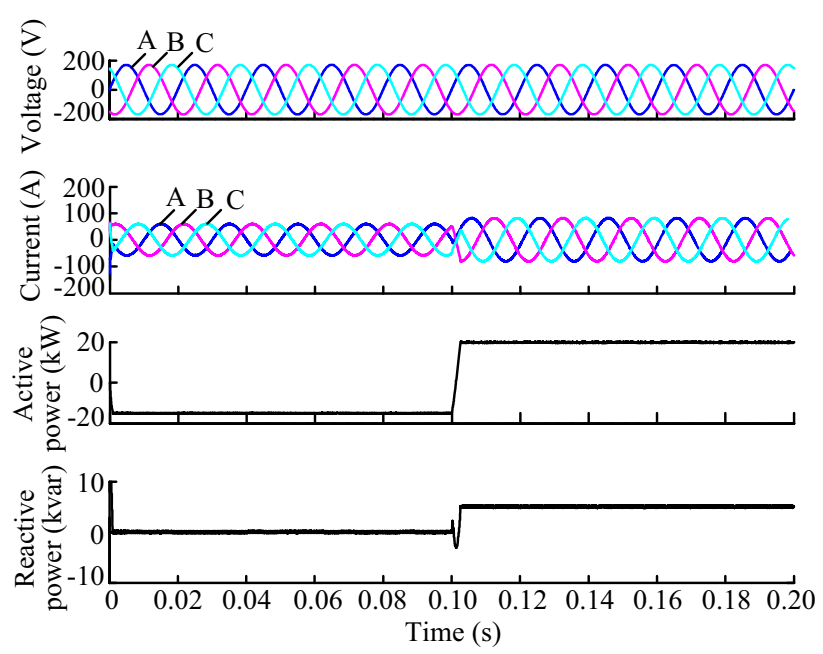

Fig. 18 System response in case 6 


\section{Experiments}

In this section, an experiment platform with two paralleled DERs as exhibited in Fig. 19 is tested to verify the proposed exponential-function-based droop control strategy. The experimental parameters are similar to those parameters in the above simulation, and $K=10$. The paralleled hard-switched PWM converters are digitally controlled by TMS28335 fixed-point DSP to implement the power and voltage control algorithm where switching frequency is $20 \mathrm{kHz}$.

\subsection{Experiment case 1}

The two DERs have the same power capacities $\left(S_{1}=S_{2}=5 \mathrm{kVA}\right)$, and both of them provide power for the load. It is considered that the load is located closer to DER1. At $t=0 \mathrm{~ms}$, the load is set as $P_{\text {load }}=2 \mathrm{~kW}, Q_{\text {load }}=1.2 \mathrm{kvar}$. Figures 20 and 21 show the reactive power sharing with different droop controllers in this experimental test condition. With the proposed exponential-function-based droop control, the output reactive power of DER1 and DER2 both is $0.6 \mathrm{kvar}$, as shown in Fig. 20. Nevertheless, with the conventional droop control, the output reactive power of DER1 is different from that of DER2. Further, at $t=120 \mathrm{~ms}$, the reactive power of the load increases to $2.4 \mathrm{kvar}$, and at $t=350 \mathrm{~ms}$, the reactive power of the load decreases to 1.2 kvar. Wherein, the output reactive power with the proposed droop control of DER1 and DER2 is equal, whereas the output reactive power with the conventional droop control of DER1 and DER2 is different. Thus, the proposed exponential-function-based droop control strategy can decrease the reactive power-sharing deviation among multiple DERs.

\subsection{Experiment case 2}

In this case, the two DERs have different power capacities ( $\left.S_{1}=5 \mathrm{kVA}, S_{2}=2.5 \mathrm{kVA}\right)$, and both of them provide power for the load. It is considered that the load is located closer to DER1. Therefore, the line impedances are set as $Z_{1}=0.01 \Omega$ and $Z_{2}=(1000+\mathrm{j} 20) \mathrm{m} \Omega$. At $t=0 \mathrm{~ms}$, the load is set as $P_{\text {load }}=2 \mathrm{~kW}, Q_{\text {load }}=$

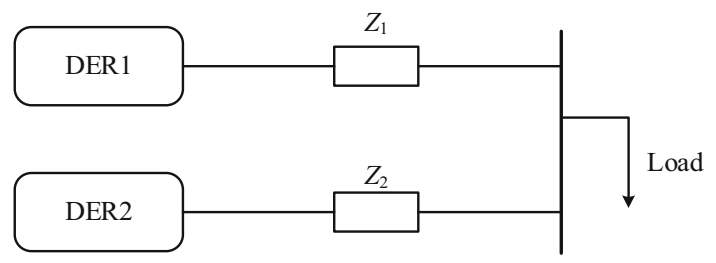

Fig. 19 Experiment platform

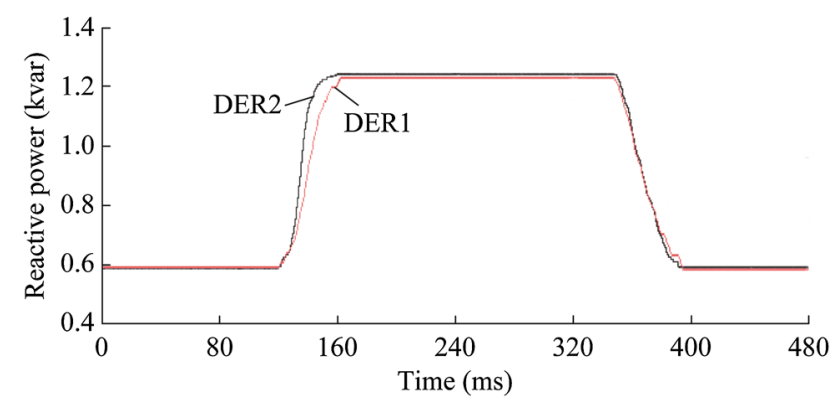

Fig. 20 Reactive power sharing with proposed droop control in experiment case 1 when DC source capacity is same

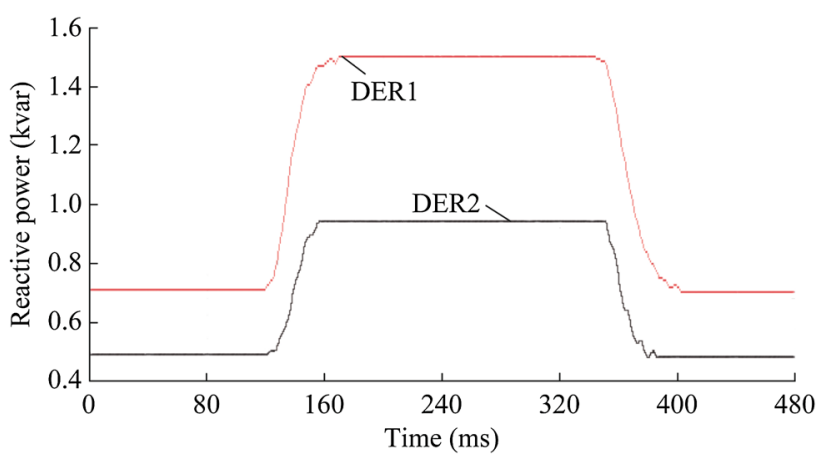

Fig. 21 Reactive power sharing with conventional droop control in experiment case 1 when DC source capacity is same

0.9 kvar. Figures 22 and 23 show the reactive power sharing with different droop controllers in this experimental test condition. With the proposed exponentialfunction-based droop control, the output reactive power of DER1 is 0.6 kvar and output reactive power of DER2 is 0.3 kvar, as shown in Fig. 22. Nevertheless, the sharing of the reactive power has obvious errors with the conventional droop control. Further, at $t=120 \mathrm{~ms}$, the reactive power of the load increases to $1.8 \mathrm{kvar}$, and at $t=350 \mathrm{~ms}$, the reactive power of the load decreases to 0.9 kvar. From Fig. 22 to Fig. 23, it is obvious that the reactive power sharing with the proposed droop control is always accurate, but the sharing of the reactive power with the conventional

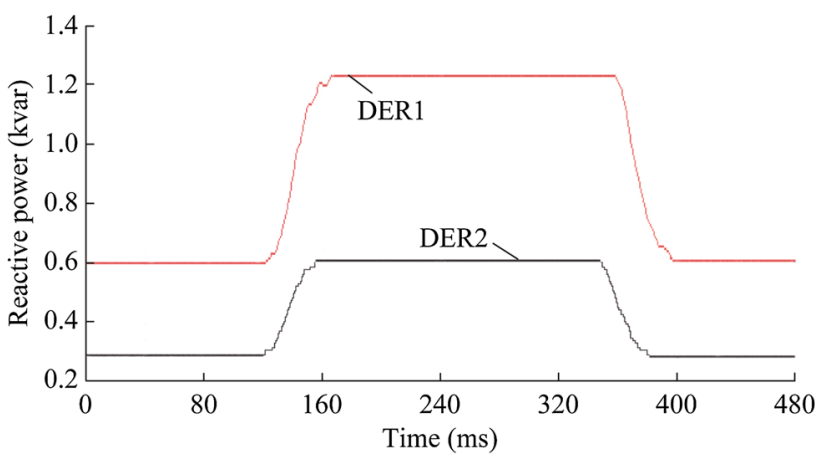

Fig. 22 Reactive power sharing with proposed droop control in experiment case 2 when DC source capacity is different 


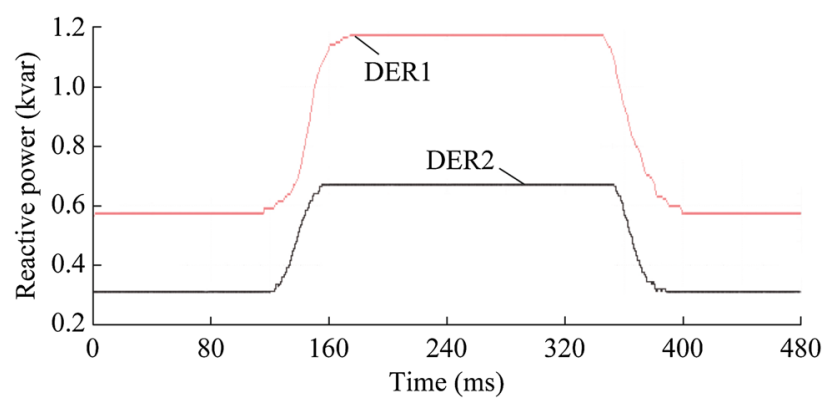

Fig. 23 Reactive power sharing with conventional droop control in experiment case 2 when DC source capacity is different

droop control has obvious errors. Thus, the proposed exponential-function-based droop control strategy is able to decrease the reactive power-sharing deviation among multiple DERs.

\section{Conclusion}

This paper proposes an exponential-function-based droop control strategy without additional communication to actualize the accurate reactive power sharing and limit the minimum value of frequency and voltage. Furthermore, the voltage and frequency can be quickly reduced to improve the utilization rate of renewable energy by the properties of exponential function when renewable energy adopts the conventional droop control strategy, and other energy employs the proposed exponential-function-based control strategy. Then, the output voltages of DERs are also identical when the rated reactive power of DERs is the same. Moreover, the proposed control strategy can also be applied to the bidirectional power flow control in the $\mathrm{AC} / \mathrm{DC}$ microgrids by measuring the AMG frequency as well as the DC bus voltage and using proposed droop characteristic. The performance of the proposed control strategy considering different operating cases has been demonstrated by the simulation of a hybrid AC/DC microgrids in the MATLAB/Simulink and experiments.

Acknowledgements This work was supported by National Key Research and Development Program of China (No. 2017YFF0108800), National Natural Science Foundation of China (Nos. 61773109, 6143304), and Major Program of National Natural Foundation of China (No. 61573094).

Open Access This article is distributed under the terms of the Creative Commons Attribution 4.0 International License (http:// creativecommons.org/licenses/by/4.0/), which permits unrestricted use, distribution, and reproduction in any medium, provided you give appropriate credit to the original author(s) and the source, provide a link to the Creative Commons license, and indicate if changes were made.

\section{References}

[1] Li Y, Zhang H, Liang X et al (2019) Event-triggered based distributed cooperative energy management for multi-energy systems. IEEE Trans Ind Inform 15(4):2008-2022

[2] Wang Z, Huang Z, Song C et al (2018) Multiscale adaptive fault diagnosis based on signal symmetry reconstitution preprocessing for microgrid inverter under changing load condition. IEEE Trans Smart Grid 9(2):797-806

[3] Sun Q, Zhang Y, He H et al (2017) A novel energy functionbased stability evaluation and nonlinear control approach for energy internet. IEEE Trans Smart Grid 8(3):1195-1210

[4] Ma D, Sun Q, Xie X et al (2018) Event triggering power sharing control for AC/DC microgrids based on P-F droop curve method. J Frankl Inst. https://doi.org/10.1016/j.jfranklin.2018. 10.025

[5] Huang Z, Wang Z, Zhang H (2018) A diagnosis algorithm for multiple open-circuited faults of microgrid inverters based on main fault component analysis. IEEE Trans Energy Convers 33(3):925-937

[6] Han R, Wang H, Jin Z et al (2018) Compromised controller design for current sharing and voltage regulation in DC microgrid. IEEE Trans Power Electron. https://doi.org/10.1109/ tpel.2018.2878084

[7] Wang R, Sun Q, Ma D et al (2019) The small-signal stability analysis of the droop-controlled converter in electromagnetic timescale. IEEE Trans Sustain Energy. https://doi.org/10.1109/ tste.2019.2894633

[8] Loh PC, Li D, Chai YK et al (2013) Autonomous operation of hybrid microgrid with AC and DC subgrids. IEEE Trans Power Electron 28(5):2214-2223

[9] Lee CT, Jiang RP, Cheng PT (2013) A grid synchronization method for droop-controlled distributed energy resource converters. IEEE Trans Ind Appl 49(2):954-962

[10] Eghtedarpour N, Farjah E (2014) Power control and management in a hybrid AC/DC microgrid. IEEE Trans Smart Grid 5(3): 1494-1505

[11] Lee CT, Chu CC, Cheng PT (2013) A new droop control method for the autonomous operation of distributed energy resource interface converters. IEEE Trans Power Electron 28(4):1980-1993

[12] Zhang H, Zhou J, Sun Q et al (2017) Data-driven control for interlinked $\mathrm{AC} / \mathrm{DC}$ microgrids via model-free adaptive control and dual-droop control. IEEE Trans Smart Grid 8(2):557-571

[13] Sun Q, Han R, Zhang H et al (2015) A multiagent-based consensus algorithm for distributed coordinated control of distributed generators in the energy internet. IEEE Trans Smart Grid 6(6):3006-3019

[14] Zhou J, Kim S, Zhang H et al (2018) Consensus-based distributed control for accurate reactive, harmonic, and imbalance power sharing in microgrids. IEEE Trans Smart Grid 9:2453-2467

[15] Chen G, Guo Z (2018) Distributed secondary and optimal active power sharing control for islanded microgrids with communication delays. IEEE Trans Smart Grid 10(2):2002-2014

[16] Rey JM, Rosero CX, Velasco M et al (2018) Local frequency restoration for droop-controlled parallel inverters in islanded microgrids. IEEE Trans Energy Convers. https://doi.org/10. 1109/tec.2018.2886267

[17] Zhang H, Kim S, Sun Q et al (2017) Distributed adaptive virtual impedance control for accurate reactive power sharing based on consensus control in microgrids. IEEE Trans Smart Grid 8(4):1749-1761 
[18] Chen X, Wang L, Sun H et al (2017) Fuzzy logic based adaptive droop control in multiterminal HVDC for wind power integration. IEEE Trans Energy Convers 32(3):1200-1208

[19] Guerrero JM, de Vicuna LG, Matas J et al (2005) Output impedance design of parallel-connected UPS inverters with wireless load-sharing control. IEEE Trans Ind Electron 52(4):1126-1135

[20] Zhong QC (2013) Robust droop controller for accurate proportional load sharing among inverters operated in parallel. IEEE Trans Ind Electron 60(4):1281-1290

[21] Zhou J, Zhang H, Sun Q et al (2018) Event-based distributed active power sharing control for interconnected AC and DC microgrids. IEEE Trans Smart Grid 9(6):6815-6828

[22] Li Y, Li YW (2011) Power management of inverter interfaced autonomous microgrid based on virtual frequency-voltage frame. IEEE Trans Smart Grid 2(1):30-40

[23] Becker C, Braun W, Carrick K et al (1994) Proposed chapter 9 for predicting voltage sags (dips) in revision to IEEE Std 493, the Gold Book. IEEE Trans Ind Appl 30(3):805-821

[24] Tian J, Zhou D, Su C et al (2017) Reactive power dispatch method in wind farms to improve the lifetime of power converter considering wake effect. IEEE Trans Sustain Energy $8(2): 477-487$

[25] Coelho EAA, Cortizo PC, Garcia PFD (2002) Small-signal stability for parallel-connected inverters in stand-alone AC supply systems. IEEE Trans Ind Appl 38(2):533-542

[26] Micallef A, Apap M, Spiteri-Staines C et al (2014) Reactive power sharing and voltage harmonic distortion compensation of droop controlled single phase islanded microgrids. IEEE Trans Smart Grid 5(3):1149-1158

[27] Yang J, Zheng W, Li S et al (2015) Design of a prediction accuracy enhanced continuous-time MPC for disturbed systems via a disturbance observer. IEEE Trans Ind Electron 62(9):5807-5816
Rui WANG received the B.S. degree in Northeastern University, Shenyang, China, in 2016. He is currently pursuing the Ph.D. degree in the School of Information Science and Engineering, Institute of Automation, Northeastern University, Shenyang, China. His current research interests include collaborative optimization of distributed generation and its stability analysis of electromagnetic timescale in Energy Internet.

Qiuye SUN received the Ph.D. degree in control theory and control engineering from Northeastern University, Shenyang, China, in 2007. $\mathrm{He}$ is currently a professor with Northeastern University. His current research interests include analysis and diagnosis of uncertain information in power distribution network, optimization analysis technology of power distribution network and network control of distributed generation system.

Yonghao GUI received the B.S. degree in automation from Northeastern University, Shenyang, China, in 2009, and the M.S. and $\mathrm{Ph} . \mathrm{D}$. degrees in electrical engineering from Hanyang University, Seoul, South Korea, in 2012 and 2017, respectively. From February 2017 to November 2018, he worked with the Department of Energy Technology, Aalborg University, Denmark, as a postdoctoral researcher. Since December 2018, he has been working with the Automation and Control Section at the Department of Electronic Systems, Aalborg University, where he is currently an assistant professor. He has been working with power converter control and control theory.

Dazhong MA received the B.S. degree in automation, and the Ph.D. degree in control theory and control engineering from Northeastern University, Shenyang, China, in 2004 and 2011, respectively. He is currently an assistant professor with Northeastern University. His current research interests include fault diagnosis, fault-tolerant controls, energy management systems, control and optimization of distributed generation systems, microgrids, and Energy Internet. 